\title{
Research Article Synchronization of Unified Chaotic Systems Using Sliding Mode Controller
}

\author{
Yi-You Hou, ${ }^{1}$ Ben-Yi Liau, ${ }^{2}$ and Hsin-Chieh Chen ${ }^{2}$ \\ ${ }^{1}$ Department of Electrical Engineering, Far East University, Tainan 74448, Taiwan \\ ${ }^{2}$ Department of Biomedical Engineering, Hungkuang University, Taichung 43302, Taiwan
}

Correspondence should be addressed to Hsin-Chieh Chen, hcchen@sunrise.hk.edu.tw

Received 29 September 2012; Accepted 21 November 2012

Academic Editor: Teh-Lu Liao

Copyright (c) 2012 Yi-You Hou et al. This is an open access article distributed under the Creative Commons Attribution License, which permits unrestricted use, distribution, and reproduction in any medium, provided the original work is properly cited.

This paper presents a method for synchronizing the unified chaotic systems via a sliding mode controller (SMC). The unified chaotic system and problem formulation are described. Two identical unified chaotic systems can be synchronized using the SMC technique. The switching surface and its controller design are developed in detail. Simulation results show the feasibility of a chaotic secure communication system based on the synchronization of the Lorenz circuits via the proposed SMC.

\section{Introduction}

Chaos theory is an extensively studied branch of the theory of nonlinear systems. Lorenz presented the first well-known chaotic system, which was a third-order autonomous system with only two multiplication-type quadratic terms but displayed very complex dynamical behaviors [1]. A chaotic system is a very complex, dynamical nonlinear system whose response has intrinsic characteristics such as broadband noise-like waveforms, difficult predictability, and sensitivity to initial condition variations $[2,3]$. These properties are advantageous in secure communication systems. Therefore, the synchronization of chaotic circuits for secure communication has received a lot of research attention [4-7]. Studies have shown that it is possible to set up a chaotic communication system to obtain secure communication [8].

The synchronization between master (transmitter) and slave (receiver) chaotic systems has potential applications for secure communication [9-13]. Several control schemes have been developed for the synchronization of chaotic systems. Sliding mode control is a popular nonlinear control strategy [14-19]. For sliding mode controller (SMC) design, the Lyaponov stability method is applied to keep the nonlinear system under control. The sliding mode approach transforms a higher-order system into a lower-order system, allowing a 


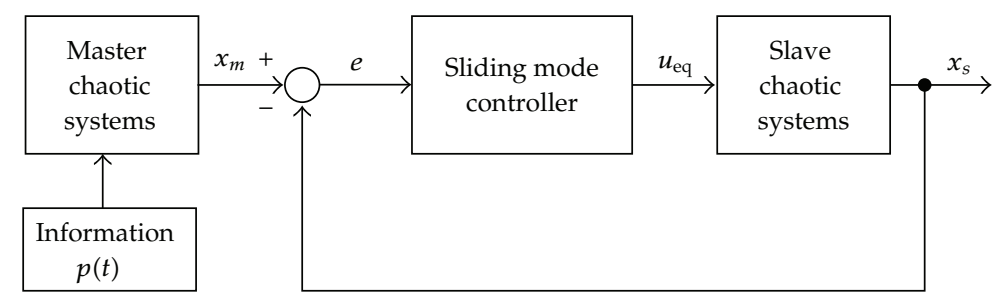

Figure 1: Block diagram of SMC-based synchronized chaotic systems.

simple control algorithm to be applied, making the system very straightforward and robust. Sliding mode control has been applied to the synchronization of chaotic systems $[5,6,11]$.

The present study designs an SMC-based chaotic secure communication system. To achieve this goal, a proportional-integral (PI) switching surface is first designed for the considered error dynamics system in sliding motion, and then, based on it, a sliding mode controller is derived. This controller is effective and guarantees both the occurrence of sliding motion and synchronization of the master-slave unified chaotic systems. Finally, an example is given to illustrate the usefulness of the proposed SMC.

\section{Problem Formulation and Main Results}

A sliding mode control system consisting of master and slave chaotic systems and information $p(t)$ is shown in Figure 1.

Consider the following unified chaotic system:

$$
\begin{gathered}
\dot{x}_{1}=(25 \alpha+10)\left(x_{2}-x_{1}\right) \\
\dot{x}_{2}=(28-35 \alpha) x_{1}+(29 \alpha-1) x_{2}-x_{1} x_{3} \\
\dot{x}_{3}=x_{1} x_{2}-\frac{(8+\alpha)}{3} x_{3},
\end{gathered}
$$

where $x_{1}, x_{2}$, and $x_{3}$ are states of system (2.1) and $\alpha \in[0,1]$. Obviously, the system (2.1) becomes the original Lorenz system for $\alpha=0$, while the system (2.1) becomes the original Chen system for $\alpha=1$. When $\alpha=4 / 5$, the system (2.1) becomes the critical system. In particular, the system bridges the gap between Lorenz system and Chen system. Moreover, the system is always chaotic in the whole interval $\alpha \in[0,1]$. Before the secure communication system can be constructed, the synchronization problem of the system based on the sliding mode control must first be solved. For the unified chaotic system, the master and slave systems are defined as

$$
\begin{gathered}
\dot{x}_{m 1}(t)=(25 \alpha+10)\left(x_{m 2}(t)-x_{m 1}(t)\right) \\
\dot{x}_{m 2}(t)=(28-35 \alpha) x_{m 1}(t)+(29 \alpha-1) x_{m 2}(t)-x_{m 1}(t) x_{m 3}(t)+p(t) \\
\dot{x}_{m 3}(t)=x_{m 1}(t) x_{m 2}(t)-\frac{(8+\alpha)}{3} x_{m 3}(t), \\
\dot{x}_{s 1}(t)=(25 \alpha+10)\left(x_{s 2}(t)-x_{s 1}(t)\right) \\
\dot{x}_{s 2}(t)=(28-35 \alpha) x_{s 1}(t)+(29 \alpha-1) x_{s 2}(t)-x_{m 1}(t) x_{s 3}(t)+u(t) \\
\dot{x}_{s 3}(t)=x_{m 1}(t) x_{s 2}(t)-\frac{(8+\alpha)}{3} x_{s 3}(t),
\end{gathered}
$$


where $u(t)$ is the controller output used to synchronize the master and slave systems (2.2), and $p(t)$ is the embedded message bounded by

$$
|p(t)| \leq \psi, \quad \psi>0
$$

The control goal is for the two unified chaotic systems (2.2) to be synchronized such that the resulting error vector satisfies

$$
\lim _{t \rightarrow \infty}\|e(t)\|=\lim _{t \rightarrow \infty}\left\|x_{m i}(t)-x_{s i}(t)\right\|=0, \quad i=1,2,3
$$

The error vectors and error dynamics are defined as

$$
\begin{aligned}
& e_{1}(t)=x_{m 1}(t)-x_{s 1}(t) \\
& e_{2}(t)=x_{m 2}(t)-x_{s 2}(t) \\
& e_{3}(t)=x_{m 3}(t)-x_{s 3}(t), \\
& \dot{e}_{1}(t)=\dot{x}_{m 1}(t)-\dot{x}_{s 1}(t) \\
& \dot{e}_{2}(t)=\dot{x}_{m 2}(t)-\dot{x}_{s 2}(t) \\
& \dot{e}_{3}(t)=\dot{x}_{m 3}(t)-\dot{x}_{s 3}(t) .
\end{aligned}
$$

Then, the following error dynamics are obtained:

$$
\begin{gathered}
\dot{e}_{1}(t)=(25 \alpha+10)\left(e_{2}(t)-e_{1}(t)\right) \\
\dot{e}_{2}(t)=(28-35 \alpha) e_{1}(t)+(29 \alpha-1) e_{2}(t)-x_{m 1} e_{3}(t)+p(t)-u(t) \\
\dot{e}_{3}(t)=x_{m 1} e_{2}(t)-\frac{(8+\alpha)}{3} e_{3}(t) .
\end{gathered}
$$

Therefore, an SMC must be designed such that the resulting error vector satisfies

$$
\lim _{t \rightarrow \infty}\|E(t)\|=\lim _{t \rightarrow \infty}\left\|\left[e_{1}(t) e_{2}(t) e_{3}(t)\right]\right\| \longrightarrow 0
$$

To stabilize the error dynamics (2.6) and achieve synchronization, two basic steps are used: first, an appropriate switching surface is selected such that the sliding motion on the sliding manifold is stable and ensures $\lim _{(t \rightarrow \infty)}\|E(t)\|=0$; second, an SMC law which guarantees the existence of the sliding mode $s(t)=0$ is established. To guarantee the asymptotic stability of the sliding mode, the PI switching surface $s(t)$ is defined as

$$
s(t)=e_{2}(t)+\int_{0}^{t}\left((25 \alpha+10) e_{1}(\tau)+x_{m 1} e_{3}(\tau)+\beta e_{2}(\tau)\right) d \tau
$$


where $\beta>0$ is given. It is well known that when the system operates in the sliding mode, the following equation must be satisfied $[20,21]$ :

$$
\begin{aligned}
s(t) & =e_{2}(t)+\int_{0}^{t}\left((25 \alpha+10) e_{1}(\tau)+x_{m 1} e_{3}(\tau)+\beta e_{2}(\tau)\right) d \tau \\
& =0 .
\end{aligned}
$$

Since $s(t)=0$, we consequently have

$$
\begin{aligned}
\dot{s}(t) & =\dot{e}_{2}(t)+\left((25 \alpha+10) e_{1}(t)+x_{m 1} e_{3}(\tau)+\beta e_{2}(t)\right) \\
& =0 .
\end{aligned}
$$

From (2.10), the following is obtained:

$$
\dot{e}_{2}(t)=-\left((25 \alpha+10) e_{1}(t)+x_{m 1} e_{3}(t)+\beta e_{2}(t)\right)
$$

Then, the equivalent sliding mode dynamics is obtained as

$$
\begin{gathered}
\dot{e}_{1}(t)=(25 \alpha+10)\left(e_{2}(t)-e_{1}(t)\right) \\
\dot{e}_{2}(t)=-(25 \alpha+10) e_{1}(t)+x_{m 1} e_{3}(t)+\beta e_{2}(t) \\
\dot{e}_{3}(t)=x_{m 1} e_{2}(t)-\frac{(8+\alpha)}{3} e_{3}(t) .
\end{gathered}
$$

The stability of the sliding mode dynamics (2.11) is analyzed below based on the Lyapunov stability theory.

The Lyapunov function is selected as $V=0.5\left(e_{1}^{2}(t)+e_{2}^{2}(t)+e_{3}^{2}(t)\right)$, which leads to

$$
\begin{aligned}
\dot{V}(t)= & e_{1}(t) \dot{e}_{1}(t)+e_{2}(t) \dot{e}_{2}(t)+e_{3}(t) \dot{e}_{3}(t) \\
= & (25 \alpha+10)\left(e_{2}(t)-e_{1}(t)\right) e_{1}(t) \\
& -\left((25 \alpha+10) e_{1}(t)+x_{m 1} e_{3}(t)+\beta e_{2}(t)\right) e_{2}(t) \\
& +\left(x_{m 1} e_{2}(t)-\frac{(8+\alpha)}{3} e_{3}(t)\right) e_{3}(t) \\
= & -(25 \alpha+10) e_{1}^{2}(t)-\beta e_{2}^{2}(t)-\frac{(8+\alpha)}{3} e_{3}^{2}(t)
\end{aligned}
$$

$\leq 0$.

According to Lyapunov stability theory, the sliding motion on the sliding manifold is stable and ensures $\lim _{t \rightarrow \infty}\|E(t)\|=\lim _{t \rightarrow \infty}\left\|\left[e_{1}(t) e_{2}(t) e_{3}(t)\right]\right\|=0$. 
Having established the appropriate switching surface (2.8), as described above, the next step is to design an SMC scheme to drive the system trajectories onto the sliding mode $s(t)=0$. This study proposes the following SMC:

$$
u(t)=u_{1}(t)+\eta \psi[\operatorname{sign}(s(t))], \quad \eta>1,
$$

where $u_{1}(t)=(38+10 \alpha) e_{1}(t)+(29 \alpha-1+\beta) e_{2}(t)$.

Before the scheme of the controller is given, the reaching condition of the sliding mode is derived below.

\section{Main Theorem}

Consider the error dynamics (2.6). If this system is controlled by $u(t)$ in (2.14), then the system trajectory converges to the sliding surface $s(t)=0$ and satisfies $\lim _{t \rightarrow \infty}\|E(t)\|=$ $\lim _{t \rightarrow \infty}\left\|\left[e_{1}(t) e_{2}(t) e_{3}(t)\right]\right\|=0$.

Proof. Consider the following Lyapunov function candidate:

$$
V(t)=0.5 s^{2}(t)
$$

Taking the derivative of (2.15) with respect to time and using (2.11), (2.14), and (2.15) yields

$$
\begin{aligned}
\dot{V}(t) & =s(t) \dot{s}(t) \\
& =s(t)\left(\dot{e}_{2}(t)+(25 \alpha+10) e_{1}(t)+x_{m 1} e_{3}(t)+\beta e_{2}(t)\right) \\
& \leq|s(t)| \psi-s(t) u(t) .
\end{aligned}
$$

Since $\psi>0$ and $\eta>1, \dot{V}(t)=s(t) \dot{s}(t)<0$ can be derived when $s \neq 0$. Thus, according to Lyapunov stability theory, $s(t)$ always converges to the switching surface $s=0$. Furthermore, since the error dynamics in the sliding manifold is asymptotically stable according to the discussion above, the error dynamic response of $\lim _{t \rightarrow \infty}\|E(t)\|=\lim _{t \rightarrow \infty}\left\|\left[e_{1}(t) e_{2}(t) e_{3}(t)\right]\right\|=$ 0 is satisfied. Hence, the proof is achieved completely.

After the SMC is designed to ensure $\lim _{t \rightarrow \infty}\|E(t)\|=\lim _{t \rightarrow \infty}\left\|\left[e_{1}(t) e_{2}(t) e_{3}(t)\right]\right\|=0$, then

$$
\begin{array}{cc}
\dot{e}_{1}(t)=(25 \alpha+10)\left(e_{2}(t)-e_{1}(t)\right)=0 & \\
\dot{e}_{2}(t)=(28-35 \alpha) e_{1}(t)+(29 \alpha-1) e_{2}(t) & \dot{e}_{1}(t)=0 \\
-x_{m 1} e_{3}(t)+p(t)-u(t)=0 & \Longrightarrow \\
\dot{e}_{3}(t)=x_{m 1} e_{2}(t)-\frac{(8+\alpha)}{3} e_{3}(t)=0 & \dot{e}_{2}(t)=0+p(t)-u(t)=0 \\
\dot{e}_{3}(t)=0 .
\end{array}
$$

It can be inferred that

$$
\lim _{t \rightarrow \infty}(p(t)-u(t))=0
$$


which means that the message $p(t)$ can be approximated by the control $u(t)$. From previous studies $[22,23]$, the control input $u(t)$ can be approximated by the following continuous equivalent control $u_{\text {eq }}(t)$ :

$$
u_{\mathrm{eq}}(t)=u_{1}(t)+\eta \psi\left[\frac{s(t)}{|s(t)|+\sigma}\right]
$$

where $\sigma$ is an arbitrarily small positive constant. When $\sigma$ is sufficiently small, then (2.19) will arbitrarily approach (2.14) and input message $p(t)$ can be recovered using (2.19).

\section{Numerical Simulation and Analysis}

In this section, the continuous equivalent control $u_{\mathrm{eq}}(t)$ (2.19) is utilized for the synchronization of unified chaotic circuits (2.1). When $\alpha=0$, the system becomes the original Lorenz system as

$$
\begin{gathered}
\dot{x}_{1}=10\left(x_{2}-x_{1}\right) \\
\dot{x}_{2}=28 x_{1}-x_{2}-x_{1} x_{3} \\
\dot{x}_{3}=x_{1} x_{2}-\frac{8}{3} x_{3} .
\end{gathered}
$$

As mentioned in [24], when directly implementing nonlinear Lorenz systems with electronic circuits, the major difficulty is that the state variables of the system (3.1) occupy a wide dynamic range with values that exceed reasonable power supply limits. However, this difficulty can be eliminated by a simple transformation of variables. Let the Lorenz equations be transformed into

$$
\begin{gathered}
\dot{x}_{m 1}=10\left(x_{m 2}-x_{m 1}\right) \\
\dot{x}_{m 2}=28 x_{m 1}-x_{m 2}-k_{1} x_{m 1} x_{m 3} \\
\dot{x}_{m 3}=k_{1} x_{m 1} x_{m 2}-\frac{8}{3} x_{m 3} .
\end{gathered}
$$

The system (3.2) with $k_{1}=10$, which is referred to as the transmitter, can be more easily implemented because the state variables never exceed the range of typical power supply limits.

Now consider the following Lorenz circuits:

Master Lorenz circuit as

$$
\begin{gathered}
\dot{x}_{m 1}(t)=10\left(x_{m 2}(t)-x_{m 1}(t)\right) \\
\dot{x}_{m 2}(t)=28 x_{m 1}(t)-x_{m 2}(t)-10 x_{m 1}(t) x_{m 3}(t)+p(t) \\
\dot{x}_{m 3}(t)=10 x_{m 1}(t) x_{m 2}(t)-\frac{8}{3} x_{m 3}(t),
\end{gathered}
$$




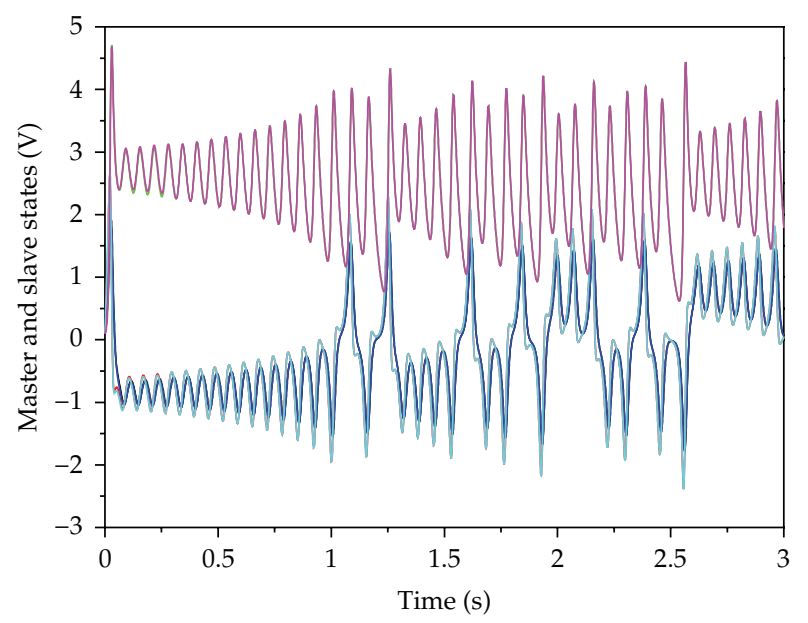

Figure 2: States of master and slave systems.

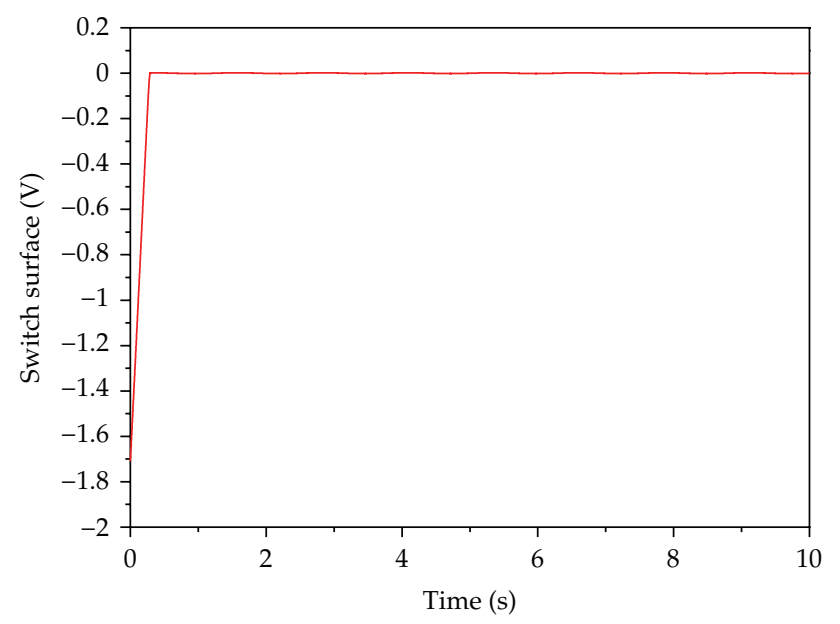

Figure 3: Simulation results of switch surface $s(t)$.

Slave Lorenz circuit as

$$
\begin{gathered}
\dot{x}_{s 1}(t)=10\left(x_{s 2}(t)-x_{s 1}(t)\right) \\
\dot{x}_{s 2}(t)=28 x_{s 1}(t)-x_{s 2}(t)-10 x_{m 1}(t) x_{s 3}(t)+u(t) \\
\dot{x}_{s 3}(t)=10 x_{m 1}(t) x_{s 1}(t) x_{s 2}(t)-\frac{8}{3} x_{s 3}(t),
\end{gathered}
$$

where $\dot{x}_{m}$ and $\dot{x}_{s}$ denote the derivatives of $x_{m}$ and $x_{s}$ with respect to time $t$, respectively. The input message $p(t)$ is a sine wave $(0.5 \mathrm{~V}, 5 \mathrm{~Hz})$ embedded into the chaotic transmitter and the equivalent SMC synchronization scheme (2.9) is given in the receiver. The initial value conditions $\left[x_{m 1}(0), x_{m 2}(0), x_{m 3}(0)\right]=\left[\begin{array}{lll}0.4 & 0.1 & 0.2\end{array}\right]$ and $\left[x_{s 1}(0), x_{s 2}(0), x_{s 3}(0)\right]=\left[\begin{array}{lll}0.1 & 0.1 & 0.1\end{array}\right]$ are used in this example. The control toolbox of MATLAB was used to simulate the proposed 


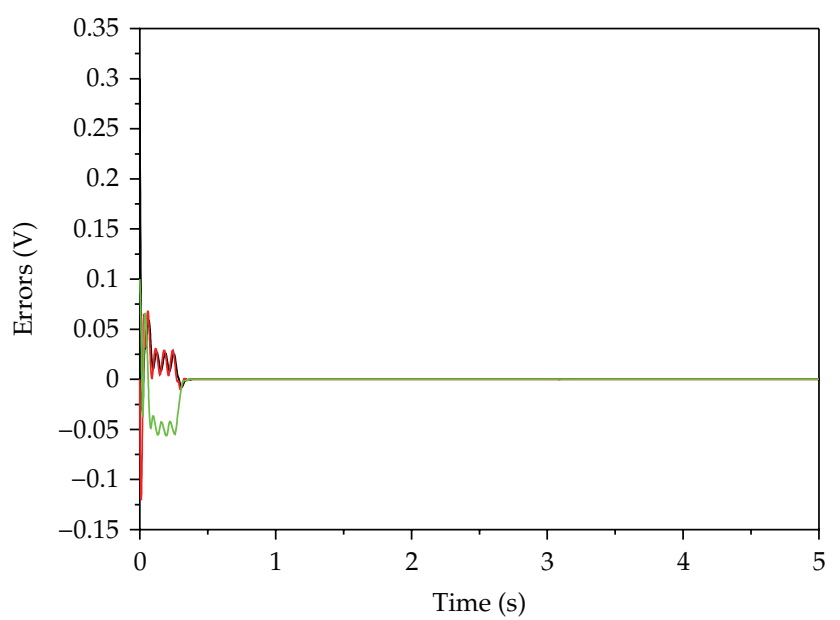

Figure 4: Simulation results of errors between state $x_{m}$ and state $x_{s}$.

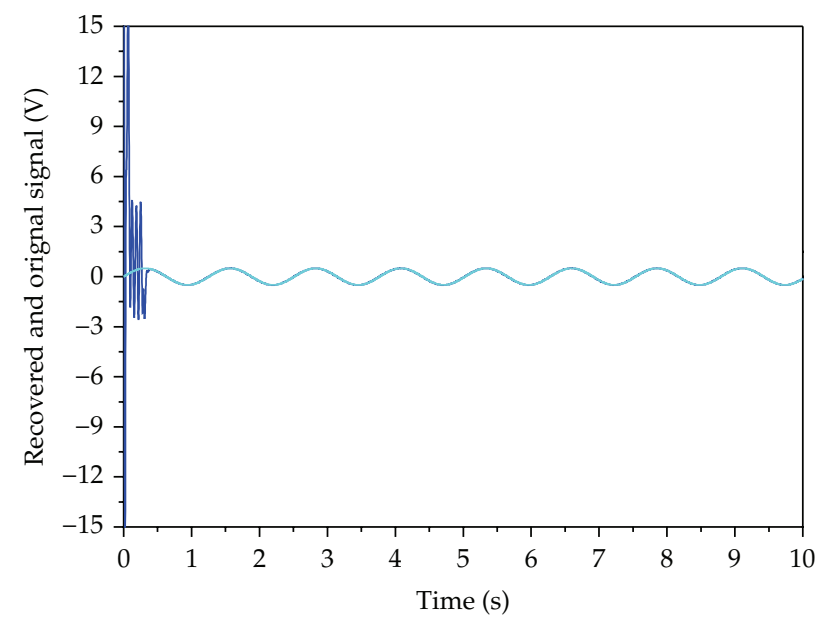

Figure 5: Simulation results of continuous equivalent control $u_{\mathrm{eq}}(t)$ and input message $p(t)$.

secure commutation system. Figure 2 shows the states of the master and slave systems. Figure 3 shows the simulation results of the switch surface $s(t)$. Figure 4 shows the simulation results of errors between state $x_{m}$ and state $x_{s}$. These figures show that the switching surface $s(t)$ converges to almost zero within $8 \mathrm{~s}$ and that the synchronization errors converge to almost zero after $0.5 \mathrm{~s}$. The master and slave are then synchronous. Figure 5 shows the simulation results of the continuous equivalent control $u_{\mathrm{eq}}(t)$ and input message $p(t)$. The input message $p(t)$ can be recovered.

\section{Conclusion}

This study presented a method for synchronizing the unified chaotic systems via an SMC. The simulation results show the feasibility of the chaotic secure communication system based on the synchronization of the Lorenz circuits via an SMC. 


\section{Acknowledgments}

The authors thank the National Science Council of Taiwan for supporting this work under Grants NSC 101-2622-E-269-012-CC3 and NSC 101-2221-E-241-002. The authors also wish to thank the anonymous reviewers for providing constructive suggestions.

\section{References}

[1] E. N. Lorenz, "Deterministic non-periodic flows," Journal of Atmospheric Sciences, vol. 20, pp. 130-141, 1963.

[2] L. M. Pecora and T. L. Carroll, "Synchronization in chaotic systems," Physical Review Letters, vol. 64, no. 8, pp. 821-824, 1990.

[3] C. K. Huang, S. C. Tsay, and Y. R. Wu, "Implementation of chaotic secure communication systems based on OPA circuits," Chaos, Solitons and Fractals, vol. 23, no. 2, pp. 589-600, 2005.

[4] G. Alvarez, L. Hernández, J. Muñoz, F. Montoya, and S. Li, "Security analysis of communication system based on the synchronization of different order chaotic systems," Physics Letters A, vol. 345, no. 4-6, pp. 245-250, 2005.

[5] H. T. Yau, C. L. Kuo, and J. J. Yan, "Fuzzy sliding mode control for a class of chaos synchronization with uncertainties," International Journal of Nonlinear Sciences and Numerical Simulation, vol. 7, no. 3, pp. 333-338, 2006.

[6] R. Martinez-Guerra and W. Yu, "Chaotic synchronization and secure communication via slidingmode observer," International Journal of Bifurcation and Chaos, vol. 18, no. 1, pp. 235-243, 2008.

[7] M. Hu and Z. Xu, "Adaptive feedback controller for projective synchronization," Nonlinear Analysis: Real World Applications, vol. 9, no. 3, pp. 1253-1260, 2008.

[8] T. L. Liao and S. H. Tsai, "Adaptive synchronization of chaotic systems and its application to secure communications," Chaos, Solitons and Fractals, vol. 11, no. 9, pp. 1387-1396, 2000.

[9] Z. Li, K. Li, C. Wen, and Y. C. Soh, "A new chaotic secure communication system," IEEE Transactions on Communications, vol. 51, no. 8, pp. 1306-1312, 2003.

[10] Z. Li and D. Xu, "A secure communication scheme using projective chaos synchronization," Chaos, Solitons and Fractals, vol. 22, no. 2, pp. 477-481, 2004.

[11] D. I. R. Almeida, J. Alvarez, and J. G. Barajas, "Robust synchronization of Sprott circuits using sliding mode control," Chaos, Solitons and Fractals, vol. 30, no. 1, pp. 11-18, 2006.

[12] J. Zhou, H. B. Huang, G. X. Qi, P. Yang, and X. Xie, "Communication with spatial periodic chaos synchronization," Physics Letters A, vol. 335, no. 2-3, pp. 191-196, 2005.

[13] G. Wen, Q. G. Wang, C. Lin, X. Han, and G. Li, "Synthesis for robust synchronization of chaotic systems under output feedback control with multiple random delays," Chaos, Solitons and Fractals, vol. 29, no. 5, pp. 1142-1146, 2006.

[14] M. M. El-Dessoky and M. T. Yassen, "Adaptive feedback control for chaos control and synchronization for new chaotic dynamical system," Mathematical Problems in Engineering, vol. 2012, Article ID 347210, 12 pages, 2012.

[15] Y. Yu and H. X. Li, "Adaptive hybrid projective synchronization of uncertain chaotic systems based on backstepping design," Nonlinear Analysis: Real World Applications, vol. 12, no. 1, pp. 388-393, 2011.

[16] C. Zhu, "Feedback control methods for stabilizing unstable equilibrium points in a new chaotic system," Nonlinear Analysis: Theory, Methods and Applications, vol. 71, no. 7-8, pp. 2441-2446, 2009.

[17] J. S. Lin and J. J. Yan, "Adaptive synchronization for two identical generalized Lorenz chaotic systems via a single controller," Nonlinear Analysis: Real World Applications, vol. 10, no. 2, pp. 1151-1159, 2009.

[18] H. Zhu and B. Cui, "Stabilization and synchronization of chaotic systems via intermittent control," Communications in Nonlinear Science and Numerical Simulation, vol. 15, no. 11, pp. 3577-3586, 2010.

[19] H. T. Yau and C. S. Shieh, "Chaos synchronization using fuzzy logic controller," Nonlinear Analysis: Real World Applications, vol. 9, no. 4, pp. 1800-1810, 2008.

[20] U. Itkis, Control System of Variable Structure, Wiley, New York, NY, USA, 1976.

[21] V. I. Utkin, Sliding Mode and Their Application in Variable Structure Systems, Mir Editors, Moscow, Russia, 1978.

[22] C. P. Tan and C. Edwards, "Sliding mode observers for detection and reconstruction of sensor faults," Automatica, vol. 38, no. 10, pp. 1815-1821, 2002.

[23] C. Edwards, S. K. Spurgeon, and R. J. Patton, "Sliding mode observers for fault detection and isolation," Automatica, vol. 36, no. 4, pp. 541-553, 2000. 
[24] K. M. Cuomo, A. V. Oppenheim, and S. H. Strogatz, "Synchronization of Lorenz-based chaotic circuits with applications to communications," IEEE Transactions on Circuits and Systems II, vol. 40, no. 10, pp. 626-633, 1993. 


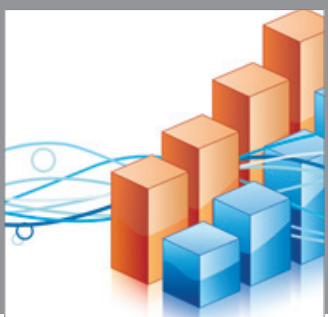

Advances in

Operations Research

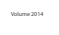

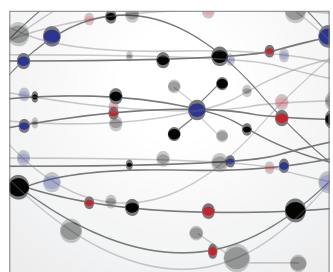

\section{The Scientific} World Journal
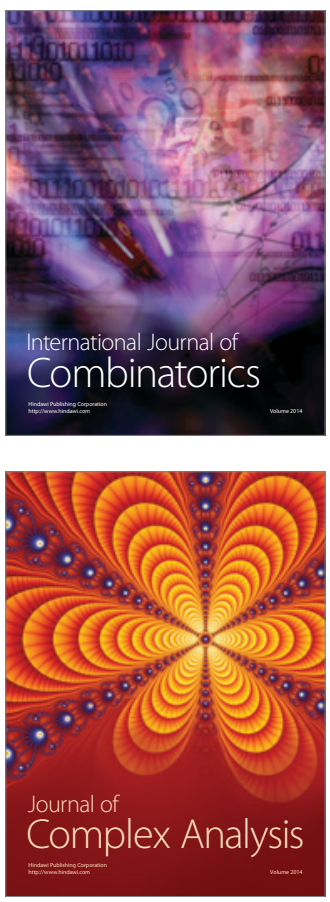

International Journal of

Mathematics and

Mathematical

Sciences
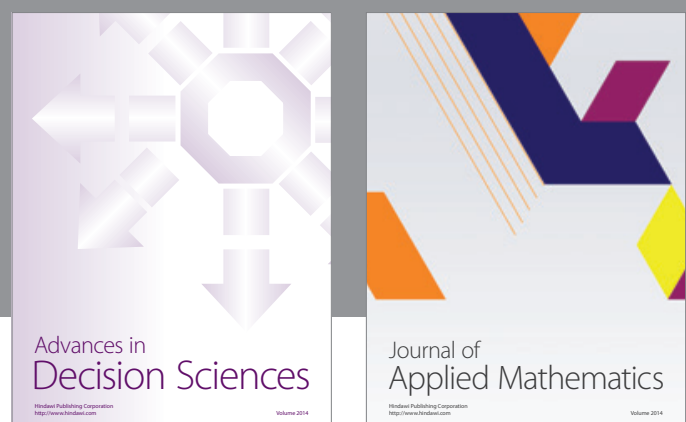

Journal of

Applied Mathematics
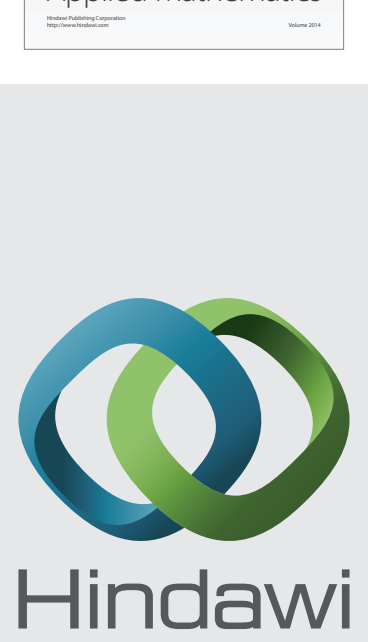

Submit your manuscripts at http://www.hindawi.com
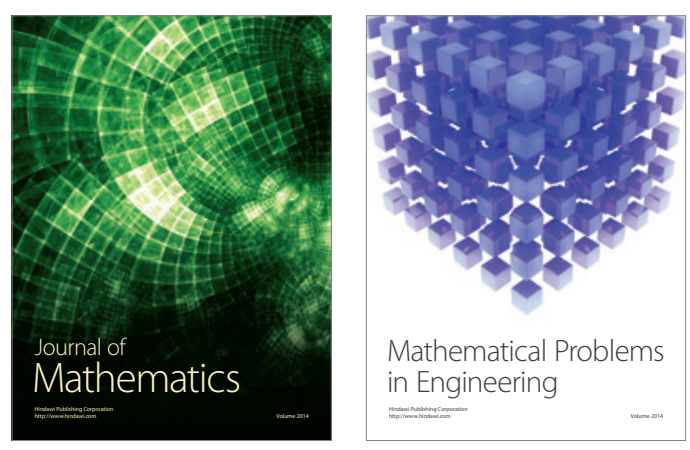

Mathematical Problems in Engineering
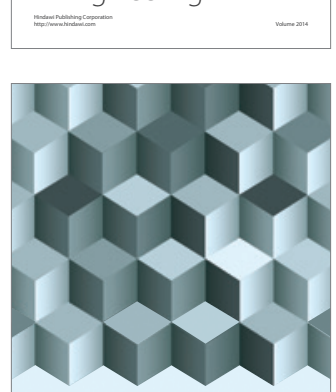

Journal of

Function Spaces
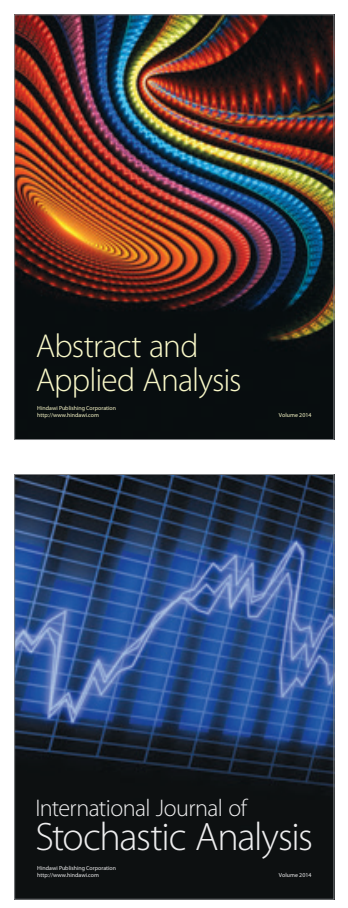

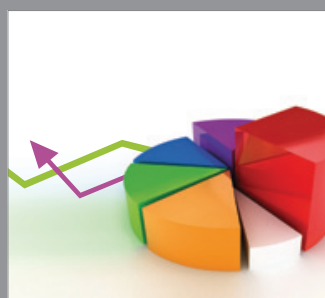

ournal of

Probability and Statistics

Promensencen
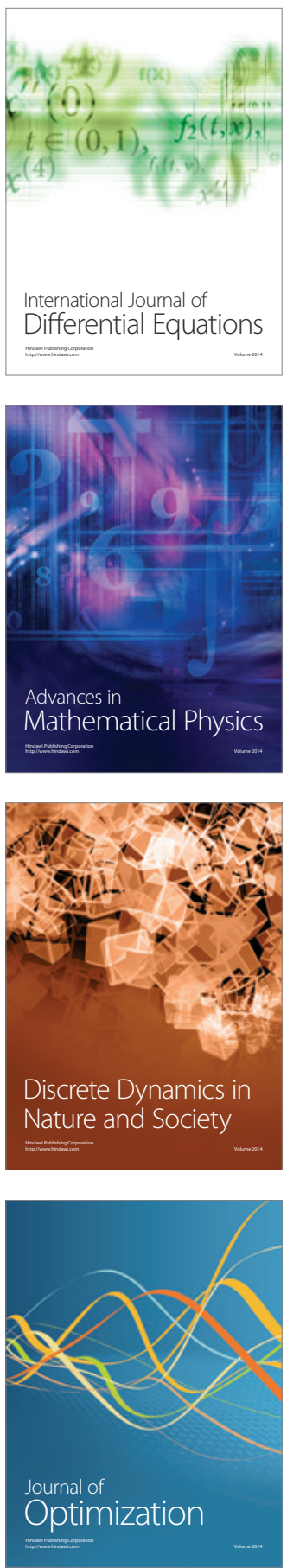